\title{
Preparation of (2R)-2-acetoxy-D-forosamine for the total synthesis of spinosyns
}

\author{
Lutz F. Tietze, * Niels Böhnke, and Gordon Brasche \\ Institut für Organische und Biomolekulare Chemie, Georg-August-Universität Göttingen, \\ Tammannstraße 2, 37077 Göttingen, Germany \\ E-mail: ltietze@gwdg.de
}

Dedicated to Professor Atta-ur-Rahman on the occasion of his $65^{\text {th }}$ birthday

\begin{abstract}
A synthesis of 2-acetoxy-D-forosamine (4) starting from 1,2-O-propylidene- $\alpha$-D-abequose (3,6dideoxy-1,2-O-propylidene- $\alpha$-D-xylo-hexopyranose) (5) was developed by introduction of an azide moiety at $\mathrm{C}-4$ with inversion of configuration. The 2-acetoxy group in 4 allows a $\beta$-selective glycosidation of a secondary alcohol moiety which is necessary in the total synthesis of spinosyns.
\end{abstract}

Keywords: Forosamine, glycosidation, neighbouring group effect, trichloroacetimidates, spinosyn

\section{Introduction}

The spinosyns represent a group of chemical related metabolites which were extracted from the soil organism Saccharopolyspora spinosa in 1986 and which reveal a strong insecticidal activity (Figure 1). The compounds contain a macrocyclic lactone connected to a tricyclic backbone. ${ }^{1}$ Both the structure and the mode of action of these insecticides are unique; they bind to the $\gamma$-amino butyric acid (GABA) receptor and in addition they interact with the nicotineacetylcholine receptor (n-AChR) localized in the postsynaptic cells. This leads to an ion influx and therefore to a generally increased muscle activity which causes to the death of the insect. ${ }^{2}$ At the moment the drug is produced fermentatorically from cell cultures and is being marketed e.g. under the brands Spinosad $\AA$, Tracer ${ }^{\circledR}$ and Success ${ }^{\circledR}$ which contain a mixture of spinosyn A (1) and $D(2)$ in a ratio of 85:15. Since first signs of resistance in Thailand and Hawaii have occurred $^{3}$, new analogues of the drug have to be developed for a conscientious resistance management. 


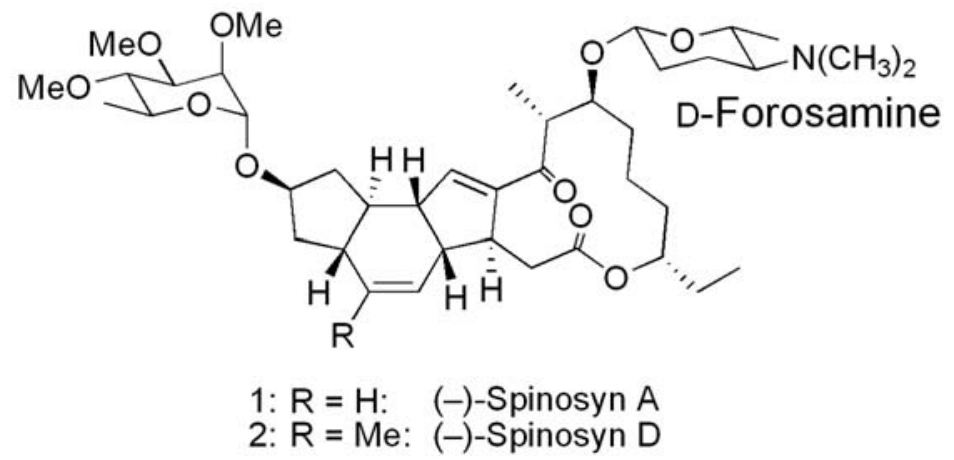

Figure 1. Structures of (-)-spinosyn A (1), (-)-spinosyn D (2).

Several syntheses of spinosyns have already been described. ${ }^{4}$ One of the main problems in these syntheses is the stereoselective introduction of the forosamine moiety, which is crucial for the bioactivity of the spinosyns. Forosamine (3) is a 2-deoxysugar which makes it difficult to control the stereochemistry of the glycosidation to give the desired $\beta$-glycoside (Scheme 1). Very recently, W. R. Roush described an efficient synthesis and highly $\beta$-selective glycosidation of a 4-azido-2-acetoxy analogue of forosamine. ${ }^{4 \mathrm{~d}}$ Besides the removal of the acetoxy group this strategy furthermore implies two additional steps: the reduction of the azide group and a dimethylation to form the forosaminyl glycoside which could be problematic with easily reducible glycosyl acceptors. It was therefore our strategy to use a 2-acetoxyforosamine (4) for a $\beta$-selective glycosidation with the benefit of the neighbouring group effect of the acetoxy group including the 4-dimethylamino group. Herein, we describe the synthesis of 2-acetoxyforosamine (4) from 1,2-O-propylidene- $\alpha$-D-abequose (3,6-dideoxy-1,2-O-propylidene- $\alpha$-D-xylo-hexopyranose $)^{5}(\mathbf{5})$ and its use in a $\beta$-selective glycosidation.

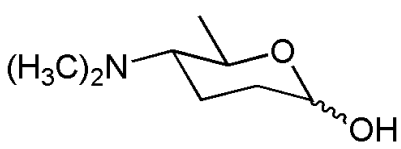

3

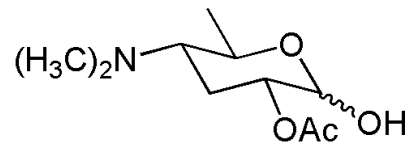

4

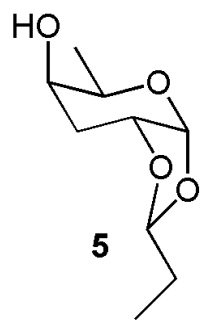

Scheme 1. Structures of forosamine (3), 2-acetoxyforosamine (4) and 1,2-O-propylidene- $\alpha$-Dabequose (5). 


\section{Results and Discussion}

Starting from literature known 1,2-O-propylidene- $\alpha$-D-abequose (5) the introduction of the equatorial amino group at C-4 called for a substitution with an $N$-nucleophile. Therefore, the axial hydroxy group at C-4 was converted into the methane- ${ }^{6}$ and the $p$-toluenesulphonate ${ }^{7} 6$ and 7, respectively; the corresponding trifluoromethanesulphonate was also prepared ${ }^{8}$, but proved to be too unstable in the following reactions using lithium dimethylamide or sodium azide in DMF at $0{ }^{\circ} \mathrm{C}$, respectively. Under the latter conditions however, 6 and 7 could easily be transformed into the corresponding azide 8. ${ }^{9}$ A comparison of the two substrates 6 and 7 revealed that the mesylate 7 was not only easier to generate but also gave the better yields in the substitution with $91 \%$ for two steps.

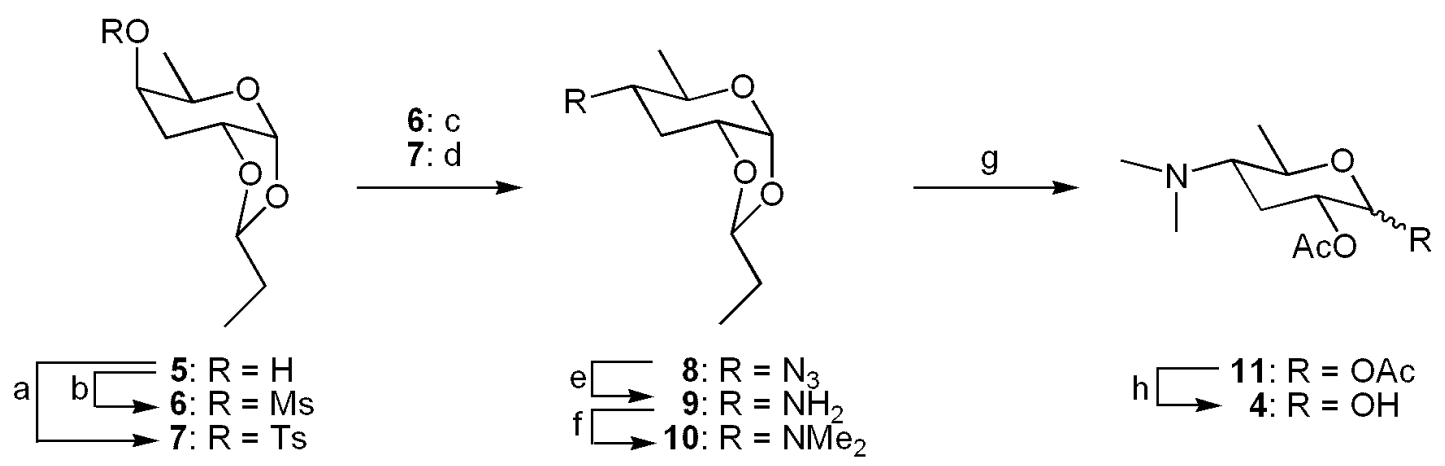

Scheme 2. (a) TsCl, DMAP, pyridine, r.t., $3 \mathrm{~h}, 50{ }^{\circ} \mathrm{C}, 20 \mathrm{~h}, 49 \%$; (b) $\mathrm{MsCl}$, pyridine, $\mathrm{CH}_{2} \mathrm{Cl}_{2}$, $0{ }^{\circ} \mathrm{C}, 5$ min, r.t., 14 h, quant.; (c) $\mathrm{NaN}_{3}$, DMF, $70{ }^{\circ} \mathrm{C}, 18 \mathrm{~h}, 65 \%$; (d) $\mathrm{NaN}_{3}$, DMSO, $85{ }^{\circ} \mathrm{C}, 14$ h, $91 \%$; (e) $\mathrm{Pd}$ (10\% on charcoal, wet), $\mathrm{H}_{2}, \mathrm{MeOH}$, r.t., 14 h, $85 \%$; (f) $\mathrm{Pd}$ (10\% on charcoal, wet), aq. $\mathrm{HCHO}(30 \%), \mathrm{MeOH}$, r.t., $14 \mathrm{~h}, 92 \%$; (g) $\mathrm{HClO}_{4}$, acetic anhydride, $-12{ }^{\circ} \mathrm{C}, 10 \mathrm{~h}$, $76 \%$; (h) ethanolamine, ethyl acetate, r.t., $3.5 \mathrm{~d}, 73 \%$.

The configuration of $\mathrm{C}-4$ in $\mathbf{8}$ could be revealed by ${ }^{1} \mathrm{H}-\mathrm{NMR}$ spectroscopy showing a doublet at $\delta=3.17$ with a coupling constant of $J_{4,5}=9.0 \mathrm{~Hz}$, which proves an equatorial orientation of the azide group.

8 was reduced to the corresponding amino compound $9,{ }^{10}$ which was transformed into the described dimethylamino compound $\mathbf{1 0}$ by a reductive amination. ${ }^{11}$ A one-pot process in which the azide $\mathbf{8}$ was treated directly with formaldehyde and palladium on charcoal under a hydrogen atmosphere in methanol did not lead to the desired compound although the reaction conditions of the single steps were comparable to the two-step process.

The following acid catalyzed opening of the 1,2-propylidene acetal moiety in $\mathbf{1 0}$ with simultaneous peracetylation turned out to be more difficult than expected. Thus, neither trifluoroacetic acid nor $p$-toluenesulphonic acid were strong enough to allow an opening of the acetal; triflic acid led to a decomposition of the substrate. The best result was obtained with 
perchloric acid at $-12{ }^{\circ} \mathrm{C}$ in acetic anhydride which gave the peracetylated sugar 11 in $76 \%$ yield. ${ }^{12}$

The final step of the synthesis of 2-acetoxyforosamine (4) was a selective deprotection of the anomeric hydroxyl group. The common procedure using hydrazinium acetate ${ }^{13}$ gave the product only in a modest yield of $33 \%$. The purification also proved to be rather difficult as the products and the acetylated hydrazine species showed similar polarities and solubilities. Reaction of 11 with benzylamine, ${ }^{14}$ methanolic ammonia and porcine pancreas lipase ${ }^{15}$, respectively did not show any conversion. On the other hand the use of sodium methoxide or potassium carbonate led to an unwanted solvolysis of both acetate moieties in $\mathbf{1 1}$ even at $0{ }^{\circ} \mathrm{C}$. The best result was obtained with ethanolamine in ethyl acetate at ambient temperature which gave the target compound 4 in $73 \%$ yield. $^{16}$

To prove the strategy for a $\beta$-selective glycosidation with the help of the neighbouring group effect of the 2-acetoxy group the trichloroacetimidate $\mathbf{1 2}$ was formed as the glycosyl donor using 5 equiv. of trichloroacetonitrile and 0.5 equiv. of polymer supported $\mathrm{DBU}^{17}$ at r.t. in $75 \%$ yield with a $\beta$ : $\alpha$-ratio of $>15: 1$ (Scheme 3 ). For the glycosidation with 12, the secondary alcohol isopropanol was used to allow a good comparison with the spinosyn aglycon. Reaction of 12 with isopropanol in the presence of $\mathrm{BF}_{3} \cdot \mathrm{OEt}_{2}$ and acetonitrile gave the glycosides 13 and 14 as an anomeric 3:1 mixture in favour of the desired $\beta$-anomer 14 in $78 \%{ }^{18}$

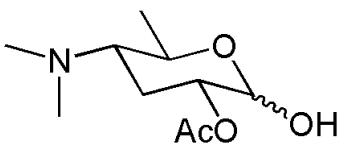

4

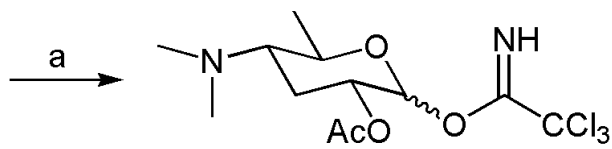

$\beta: \alpha>15: 1$

12

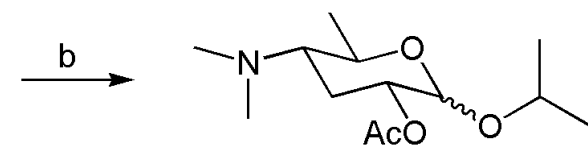

$\alpha: \beta=1: 3$

13: $\alpha$

14: $\beta$

Scheme 3. (a) $\mathrm{Cl}_{3} \mathrm{CCN}$, polymer supported $\mathrm{DBU}, \mathrm{CH}_{2} \mathrm{Cl}_{2}$, r.t., 2 h, 75 \%; (b) 6.7 equiv. iPrOH, MS $3 \AA$, 3.3 equiv. $\mathrm{BF}_{3} \cdot \mathrm{OEt}_{2}, 2.7$ eq. $\mathrm{CH}_{3} \mathrm{CN}, \mathrm{CH}_{2} \mathrm{Cl}_{2}, 0^{\circ} \mathrm{C}, 30$ min, r.t., 2.5 h, $78 \%$.

In conclusion, the aminosugar 2-acetoxyforosamine (4) could be synthesized starting from 1,2-O-propylidene- $\alpha$-D-abequose (5) in six steps with $40 \%$ overall yield. The 2 -acetoxy group in 4 allows a stereoselective formation of a $\beta$-glycoside with a secondary alcohol which is a crucial step in the synthesis of spinosyns via the intermediate formation of the trichloroacetimidate $\mathbf{1 2}$.

\section{Experimental Section}

General Procedures. Melting points were measured with a Mettler FO61 melting point apparatus and are uncorrected. Optical rotations were taken with a Perkin-Elmer 241 spectrometer. ${ }^{1} \mathrm{H}$ - and ${ }^{13} \mathrm{C}-\mathrm{NMR}$ spectra were recorded with Mercury-200, VXR-200, Unity 300, 
Inova-500, Unity Inova-600 (Varian) or AMX 300 (Bruker) spectrometers. Chemical shifts are reported in $\delta \mathrm{ppm}$ referenced to TMS $\left({ }^{1} \mathrm{H}-\mathrm{NMR}\right)$ or to $\mathrm{CDCl}_{3}\left({ }^{13} \mathrm{C} \mathrm{NMR}\right)$ as internal standard. IR spectra were taken with a Bruker Vector 22 and UV spectra with a Perkin-Elmer Lambda 2 spectrometer. Mass spectra were measured with a Varian MAT 311A (low resolution) and with a MAT 731 (high resolution). Microanalyses were performed on a CHN 2000 from LECO with the combustion unit MIKRO U/D from Heraeus. Precoated silica gel SIL G/UV254 (MachereyNagel GmbH \& Co KG) was used for TLC, and silica gel 60 (0.040-0.063 mm) (Merck KGaA) was used for flash chromatography. All reactions were performed under argon in oven-dried glassware. Solvents were dried and distilled prior to use by the usual laboratory methods, commercially available chemicals were used without further purification.

3,6-Dideoxy-4-methanesulphonyl-1,2-O-propylidene- $\alpha$-D-xylo-hexopyranose (6). To a solution of $5(700 \mathrm{mg}, 3.32 \mathrm{mmol})$ in dry dichloromethane $(14 \mathrm{~mL})$ and dry pyridine $(970 \mu \mathrm{L}$, $970 \mathrm{mg}, 12.3 \mathrm{mmol}, 3.7$ equiv.) was added dropwise with stirring at $-5{ }^{\circ} \mathrm{C}$ methanesulphonyl chloride $(770 \mu \mathrm{L}, 1.14 \mathrm{~g}, 9.96 \mathrm{mmol}, 3.0$ equiv.) and stirring continued for $14 \mathrm{~h}$ at room temperature. The mixture was diluted with dichloromethane, washed with sat. aq. $\mathrm{NaHCO}_{3}$, and the aqueous phase was extracted with dichloromethane $(3 \times 50 \mathrm{~mL})$. The combined organic phases were concentrated in vacuo after addition of toluene $(50 \mathrm{~mL})$ and the residue was purified by column chromatography ( $n$-pentane/ethyl acetate $3: 2)$ to give 6 (0.96 g, $3.32 \mathrm{mmol}$, quant.) as a colourless oil; $[\alpha]_{\mathrm{D}}^{20}=-18.4^{\circ}\left(\right.$ c 1.00 in $\left.\mathrm{CHCl}_{3}\right) ; R_{\mathrm{f}}=0.59$ (n-pentane/ethyl acetate 3:2); IR $\left(\mathrm{NaCl}, \mathrm{cm}^{-1}\right): 3026,2976,2941,2884,1356\left(\mathrm{OSO}_{2} \mathrm{CH}_{3}\right), 1175\left(\mathrm{OSO}_{2} \mathrm{CH}_{3}\right) ;{ }^{1} \mathrm{H}-\mathrm{NMR}(300 \mathrm{MHz}$, $\left.\mathrm{CDCl}_{3}\right): \delta=1.01\left(\mathrm{t}, J=7.5 \mathrm{~Hz}, 3 \mathrm{H}, 9-\mathrm{H}_{3}\right), 1.28\left(\mathrm{~d}, J=6.6 \mathrm{~Hz}, 3 \mathrm{H}, 6-\mathrm{H}_{3}\right), 1.76\left(\mathrm{~m}, 2 \mathrm{H}, 8-\mathrm{H}_{2}\right)$, 1.96 (ddd, $J=15.1,6.9,3.2 \mathrm{~Hz}, 1 \mathrm{H}, 3-\mathrm{H}_{\mathrm{a}}$ ), 2.80 (ddd, $J=15.1,8.4,3.2 \mathrm{~Hz}, 1 \mathrm{H}, 3-\mathrm{H}_{\mathrm{b}}$ ), 3.04 (s, $\left.3 \mathrm{H}, \mathrm{SO}_{2} \mathrm{CH}_{3}\right), 4.06(\mathrm{dt}, J=5.4,3.2 \mathrm{~Hz}, 1 \mathrm{H}, 2-\mathrm{H}), 4.25(\mathrm{dq}, J=6.6,4.3 \mathrm{~Hz}, 1 \mathrm{H}, 5-\mathrm{H}), 4.77(\mathrm{t}, J$ $=5.1 \mathrm{~Hz}, 1 \mathrm{H}, 7-\mathrm{H}), 4.86(\mathrm{ddd}, J=8.4,6.9,4.3 \mathrm{~Hz}, 1 \mathrm{H}, 4-\mathrm{H}), 5.43(\mathrm{~d}, J=5.3 \mathrm{~Hz}, 1 \mathrm{H}, 1-\mathrm{H})$; ${ }^{13} \mathrm{C}-\mathrm{NMR}\left(50.3 \mathrm{MHz}, \mathrm{CDCl}_{3}\right): \delta=8.37(\mathrm{C}-9), 15.55(\mathrm{C}-6), 26.48(\mathrm{C}-8), 28.97(\mathrm{C}-3), 38.37$ $\left(\mathrm{SO}_{2} \mathrm{CH}_{3}\right), 64.27$ (C-5), 71.67 (C-2), 75.56 (C-4), 96.87 (C-1), 104.51 (C-7); $\mathrm{C}_{10} \mathrm{H}_{18} \mathrm{O}_{6} \mathrm{~S}$ (266.31); HRMS-EI: Calcd. for $\mathrm{C}_{10} \mathrm{H}_{18} \mathrm{O}_{6} \mathrm{~S}: 266.0824$. Found: 266.0824.

3,6-Dideoxy-4-(p-toluenesulphonyl)-1,2-O-propylidene- $\alpha$-D-xylo-hexopyranose (7). To a solution of $5(100 \mathrm{mg}, 531 \mu \mathrm{mol})$ in dry pyridine $(5 \mathrm{~mL})$, $p$-toluenesulphonyl chloride $(506 \mathrm{mg}$, $2.66 \mathrm{mmol}, 5.0$ equiv.) and $N, N$-dimethylaminopyridine $(32.4 \mathrm{mg}, 0.266 \mathrm{mmol}, 0.5 \mathrm{equiv}$.) were added. The solution was stirred for $3 \mathrm{~h}$ at room temperature and heated to $50{ }^{\circ} \mathrm{C}$ for $20 \mathrm{~h}$. After removal of the solvent in vacuo the residue was taken up in ethyl acetate and the obtained solution washed two times with saturated aq. $\mathrm{NaHCO}_{3}$ and brine. The aqueous phases were extracted with ethyl acetate, the solution washed as described above and the combined organic phases dried over $\mathrm{MgSO}_{4}$. After removal of the solvent in vacuo the residue was purified by column chromatography ( $n$-pentane/ethyl acetate 4:1) to give $7(89.7 \mathrm{mg}, 262 \mu \mathrm{mol}, 49 \%$ ) as a colourless oil; $R_{\mathrm{f}}=0.59$ (n-pentane/ethyl acetate 3:2); ${ }^{1} \mathrm{H}-\mathrm{NMR}\left(300 \mathrm{MHz}, \mathrm{CDCl}_{3}\right): \delta=0.99$ (t, $J$ $\left.=7.7 \mathrm{~Hz}, 3 \mathrm{H}, 9-\mathrm{H}_{3}\right), 1.12\left(\mathrm{~d}, J=6.7 \mathrm{~Hz}, 3 \mathrm{H}, 6-\mathrm{H}_{3}\right), 1.67-1.78\left(\mathrm{~m}, 2 \mathrm{H}, 8-\mathrm{H}_{2}\right), 1.82(\mathrm{ddd}, J=$ 15.3, 6.9, 3.3 Hz, $1 \mathrm{H}, 3-\mathrm{H}_{\mathrm{a}}$ ), 2.46 (s, $3 \mathrm{H}, \mathrm{Ph}-\mathrm{CH}_{3}$ ), 2.52 (ddd, J=15.3, 8.4, 3.2 Hz, $1 \mathrm{H}, 3-\mathrm{H}_{\mathrm{b}}$ ), $3.96(\mathrm{dt}, J=5.3,3.2 \mathrm{~Hz}, 1 \mathrm{H}, 2-\mathrm{H}), 4.14(\mathrm{dq}, J=6.7,4.2 \mathrm{~Hz}, 1 \mathrm{H}, 5-\mathrm{H}), 4.66$ (ddd, $J=8.4,6.9$, 
$4.1 \mathrm{~Hz}, 1 \mathrm{H}, 4-\mathrm{H}), 4.73(\mathrm{t}, J=5.1 \mathrm{~Hz}, 1 \mathrm{H}, 7-\mathrm{H}), 5.37$ (d, $J=5.3 \mathrm{~Hz}, 1 \mathrm{H}, 1-\mathrm{H}), 7.35(\mathrm{~d}, J=$ $\left.8.3 \mathrm{~Hz}, 2 \mathrm{H}, 3^{\prime}-\mathrm{H}, 5^{\prime}-\mathrm{H}\right), 7.80$ (d, $\left.J=8.3 \mathrm{~Hz}, 2 \mathrm{H}, 2^{\prime}-\mathrm{H}, 66^{\prime}-\mathrm{H}\right) ;{ }^{13} \mathrm{C}-\mathrm{NMR}\left(50.3 \mathrm{MHz}, \mathrm{CDCl}_{3}\right): \delta=$

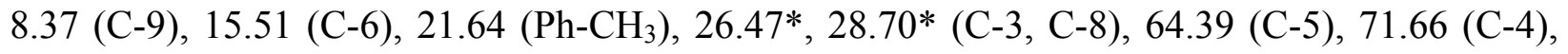
76.23 (C-2), 96.81 (C-7), 104.41 (C-1), 127.77 (C-2', C-6'), 129.85 (C-3', C-5'), 133.80 (C-1'), 144.86 (C-4'); HRMS-EI: Calcd. for $\mathrm{C}_{16} \mathrm{H}_{22} \mathrm{O}_{6} \mathrm{~S}: 342.1137$. Found: 342.1137.

(4S)-3,6-Dideoxy-4-azido-1,2-O-propylidene- $\alpha$-D-xylo-hexopyranose (8). A mixture of 6 (140 mg, $526 \mu \mathrm{mol})$ or $7(50.0 \mathrm{mg}, 146 \mu \mathrm{mol})$ and vacuum dried sodium azide (171 mg, $2.63 \mathrm{mmol}, 5.0$ equiv. or $47.5 \mathrm{mg}, 730 \mu \mathrm{mol}, 5.0$ equiv., respectively) in dry DMSO (2 mL) or DMF $(2 \mathrm{~mL})$ was heated at $85{ }^{\circ} \mathrm{C}$ for $14 \mathrm{~h}$ and at $70{ }^{\circ} \mathrm{C}$ for $18 \mathrm{~h}$, respectively. After cooling to room temperature the mixture was diluted with diethyl ether, washed with water, the aqueous phase was reextracted with diethyl ether and the combined organic phases were washed with brine and dried over $\mathrm{Na}_{2} \mathrm{SO}_{4}$. The solvent was removed under reduced pressure and the crude product purified by column chromatography (n-pentane/ethyl acetate 10:1) to give 8 (103 mg, $481 \mu \mathrm{mol}, 91 \%$ and $20.1 \mathrm{mg}, 94.0 \mu \mathrm{mol}, 65 \%$, respectively) as a colourless oil; $[\alpha]_{\mathrm{D}}^{20}=+32.5^{\circ}$ (c 1.00 in $\mathrm{CHCl}_{3}$ ), $R_{\mathrm{f}}=0.71$ (n-pentane/ethyl acetate 3:2); IR $\left(\mathrm{NaCl}, \mathrm{cm}^{-1}\right): 2975,2936,2882$, 2105 (azide); ${ }^{1} \mathrm{H}-\mathrm{NMR}\left(600 \mathrm{MHz}, \mathrm{CDCl}_{3}\right): \delta=1.01\left(\mathrm{t}, J=7.6 \mathrm{~Hz}, 3 \mathrm{H}, 9-\mathrm{H}_{3}\right), 1.28(\mathrm{~d}, J=$ $\left.6.3 \mathrm{~Hz}, 3 \mathrm{H}, 6-\mathrm{H}_{3}\right), 1.79\left(\mathrm{dq}, J=7.6,5.0 \mathrm{~Hz}, 2 \mathrm{H}, 8-\mathrm{H}_{2}\right), 2.08(\mathrm{ddd}, J=16.1,9.0,3.1 \mathrm{~Hz}, 1 \mathrm{H}$, $\left.3-\mathrm{H}_{\mathrm{a}}\right), 2.25\left(\mathrm{ddd}, J=16.1,3.0,1.4 \mathrm{~Hz}, 1 \mathrm{H}, 3-\mathrm{H}_{\mathrm{b}}\right), 3.17$ (dt, $\left.J=9.0,1.0 \mathrm{~Hz}, 1 \mathrm{H}, 4-\mathrm{H}\right), 3.82$ (dq, $J=9.0,6.3 \mathrm{~Hz}, 1 \mathrm{H}, 5-\mathrm{H}), 4.04(\mathrm{dt}, J=3.0,5.1 \mathrm{~Hz}, 1 \mathrm{H}, 2-\mathrm{H}), 4.79$ (t, $J=5.1 \mathrm{~Hz}, 1 \mathrm{H}, 7-\mathrm{H})$, $5.35(\mathrm{~d}, J=5.2 \mathrm{~Hz}, 1 \mathrm{H}, 1-\mathrm{H}) ;{ }^{13} \mathrm{C}-\mathrm{NMR}\left(50.3 \mathrm{MHz}, \mathrm{CDCl}_{3}\right): \delta=8.40(\mathrm{C}-9), 19.93(\mathrm{C}-6)$, 26.75*, 27.17* (C-3, C-8), 58.62 (C-4), 65.47 (C-5), 71.52 (C-2), 96.79 (C-1), 104.80 (C-7); MS (ESI): $\mathrm{m} / \mathrm{z}(\%)=236.1(100)[\mathrm{M}+\mathrm{Na}]^{+}$; Anal. Calcd for $\mathrm{C}_{9} \mathrm{H}_{15} \mathrm{~N}_{3} \mathrm{O}_{3}: \mathrm{C}, 50.69 ; \mathrm{H}, 7.09 ; \mathrm{N}$, 19.71; Found: C, 51.00; H, 7.09; N, 19.49.

(4S)-3,6-Dideoxy-4-amino-1,2-O-propylidene-a-D-xylo-hexopyranose (9). A solution of 8 $(447 \mathrm{mg}, 2.10 \mathrm{mmol})$ and palladium $(10 \%$ on charcoal) $(100 \mathrm{mg})$ in methanol $(25 \mathrm{~mL})$ was stirred for $14 \mathrm{~h}$ at room temperature under a hydrogen atmosphere. Then the mixture was filtered through Celite, the residue washed with methanol and the filtrate concentrated under reduced pressure. The residue was purified by column chromatography $\left(\mathrm{CH}_{2} \mathrm{Cl}_{2} /\right.$ methanol 7:1) to give 9 (335 mg, $1.79 \mathrm{mmol}, 85 \%$ ) as a colourless oil; $[\alpha]_{\mathrm{D}}^{20}=-12.5^{\circ}\left(\right.$ c 1.00 in $\left.\mathrm{CHCl}_{3}\right) ; R_{\mathrm{f}}=0.25$ $\left(\mathrm{CH}_{2} \mathrm{Cl}_{2} /\right.$ methanol 7:1); IR $\left(\mathrm{NaCl}, \mathrm{cm}^{-1}\right): 3376,3305$ (N-H st), 2971, 2932, 2881; ${ }^{1} \mathrm{H}-\mathrm{NMR}$ $\left(600 \mathrm{MHz}, \mathrm{CDCl}_{3}\right): \delta=1.00\left(\mathrm{t}, J=7.6 \mathrm{~Hz}, 3 \mathrm{H}, 9-\mathrm{H}_{3}\right), 1.28\left(\mathrm{~d}, J=6.3 \mathrm{~Hz}, 3 \mathrm{H}, 6-\mathrm{H}_{3}\right), 1.59$ (br s, $\left.2 \mathrm{H}, \mathrm{NH}_{2}\right), 1.77\left(\mathrm{~m}, 2 \mathrm{H}, 8-\mathrm{H}_{2}\right), 1.94\left(\mathrm{~m}, 1 \mathrm{H}, 3-\mathrm{H}_{\mathrm{a}}\right), 1.99$ (ddd, J = 15.1, 7.6, $2.7 \mathrm{~Hz}, 1 \mathrm{H}, 3-\mathrm{H}_{\mathrm{b}}$ ), 2.65 (dt, $J=7.6,8.2 \mathrm{~Hz}, 1 \mathrm{H}, 4-\mathrm{H}), 3.58$ (dq, $J=8.2,6.3 \mathrm{~Hz}, 1 \mathrm{H}, 5-\mathrm{H}), 4.06$ (m, $1 \mathrm{H}, 2-\mathrm{H}), 4.75$ (t, $J=5.1 \mathrm{~Hz}, 1 \mathrm{H}, 7-\mathrm{H}), 5.36(\mathrm{~d}, J=5.2 \mathrm{~Hz}, 1 \mathrm{H}, 1-\mathrm{H}) ;{ }^{13} \mathrm{C}-\mathrm{NMR}\left(150.8 \mathrm{MHz}, \mathrm{CDCl}_{3}\right): \delta=8.56$ (C-9), 19.77 (C-6), 26.85 (C-8), 30.91 (C-3), 50.37 (C-4), 70.45 (C-5), 73.38 (C-2), 97.05 (C-1), 104.40 (C-7); Anal. Calcd for $\mathrm{C}_{9} \mathrm{H}_{17} \mathrm{NO}_{3}$ : C, 57.73; H, 9.15; N, 7.48; Found: C, 57.51; H, 9.19; N, 7.33.

(4S)-3,6-Dideoxy-4-dimethylamino-1,2-O-propylidene- $\alpha$-D-xylo-hexopyranose (10). A mixture of $9(227 \mathrm{mg}, 1.62 \mathrm{mmol}), 37 \%$ formaline $(450 \mu \mathrm{L})$ and $250 \mathrm{mg}$ palladium $(10 \%$ on charcoal, wet) in methanol $(25 \mathrm{~mL})$ was stirred for $14 \mathrm{~h}$ at room temperature under a hydrogen 
atmosphere. After filtration through Celite and washing with methanol the filtrate was concentrated under reduced pressure and the residue purified by column chromatography $\left(\mathrm{CH}_{2} \mathrm{Cl}_{2} /\right.$ methanol 7:1) to give $\mathbf{1 0}(273 \mathrm{mg}, 1.27 \mathrm{mmol}, 92 \%)$ as a colourless oil; $[\alpha]_{\mathrm{D}}^{20}=+47.3^{\circ}$ (c 1.00 in $\left.\mathrm{CHCl}_{3}\right) ; R_{\mathrm{f}}=0.42\left(\mathrm{CH}_{2} \mathrm{Cl}_{2} /\right.$ methanol 7:1); IR $\left(\mathrm{NaCl}, \mathrm{cm}^{-1}\right): 2970,2935,2875,2788$ (C-H st), 1457; ${ }^{1} \mathrm{H}-\mathrm{NMR}\left(300 \mathrm{MHz}, \mathrm{CDCl}_{3}\right): \delta=1.02\left(\mathrm{t}, J=7.5 \mathrm{~Hz}, 3 \mathrm{H}, 9-\mathrm{H}_{3}\right), 1.27(\mathrm{~d}, J=$ 6.1 Hz, $\left.3 \mathrm{H}, 6-\mathrm{H}_{3}\right), 1.65-1.86\left(\mathrm{~m}, 3 \mathrm{H}, 8-\mathrm{H}_{2}, 3-\mathrm{H}_{\mathrm{a}}\right), 2.12\left(\mathrm{dt}, J=15.9,3.0 \mathrm{~Hz}, 1 \mathrm{H}, 3-\mathrm{H}_{\mathrm{b}}\right), 2.22(\mathrm{~s}$, $\left.6 \mathrm{H}, \mathrm{N}\left(\mathrm{CH}_{3}\right)_{2}\right), 2.61(\mathrm{dt}, J=10.1,3.3 \mathrm{~Hz}, 1 \mathrm{H}, 4-\mathrm{H}), 3.92(\mathrm{dq}, J=10.1,6.1 \mathrm{~Hz}, 1 \mathrm{H}, 5-\mathrm{H}), 4.04$ $(\mathrm{m}, 1 \mathrm{H}, 2-\mathrm{H}), 4.79(\mathrm{t}, J=5.0 \mathrm{~Hz}, 1 \mathrm{H}, 7-\mathrm{H}), 5.34(\mathrm{~d}, J=5.1 \mathrm{~Hz}, 1 \mathrm{H}, 1-\mathrm{H})$; ${ }^{13} \mathrm{C}-\mathrm{NMR}$

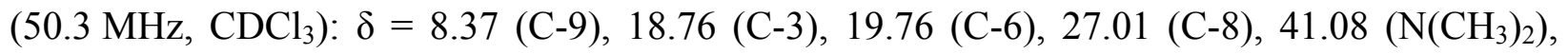
61.95 (C-4), 64.22 (C-5), 72.91 (C-2), 96.87 (C-1), 103.99 (C-7); Anal. Calcd for $\mathrm{C}_{11} \mathrm{H}_{21} \mathrm{NO}_{3}$ : C, 61.37; H, 9.83; N, 6.51; Found: C, 61.16; H, 9.87; N, 6.70.

(4S)-1,2-Di-O-acetyl-3,6-dideoxy-4-dimethylamino- $\alpha / \beta$-D-xylo-hexopyranose, (2R)-1,2-Di-Oacetyl-2-hydroxy- $\boldsymbol{\alpha} / \boldsymbol{\beta}$-D-forosamine (11). To a solution of $\mathbf{1 0}(190 \mathrm{mg}, 883 \mu \mathrm{mol})$ in acetic anhydride $(25 \mathrm{~mL})$ was added at $-12{ }^{\circ} \mathrm{C}$ with stirring $70 \%$ aq. perchloric acid $(84 \mu \mathrm{L}, 972 \mu \mathrm{mol}$, 1.1 equiv.). After $10 \mathrm{~h}$ the reaction was quenched by slow addition of cold ethanol $(25 \mathrm{ml})$ and after $30 \mathrm{~min}$ the mixture was poured onto ice, stirred for $30 \mathrm{~min}$ and neutralized with saturated aq. $\mathrm{NaHCO}_{3}$. The mixture was extracted with dichloromethane $(3 \times 50 \mathrm{~mL})$ and the combined organic phases were washed with brine and dried over $\mathrm{MgSO}_{4}$. After removing the solvent in vacuo the residue was purified by column chromatography ( $n$-pentane/ethyl acetate/methanol 7.5:2:0.5) to give $11\left(175 \mathrm{mg}, 675 \mu \mathrm{mol}, 76 \%\right.$ ) as a yellow oil; $R_{\mathrm{f}}=0.44$ (n-pentane/ethyl acetate/methanol 7:2:1); IR $\left(\mathrm{NaCl}, \mathrm{cm}^{-1}\right): 2975,2939,1748\left(\mathrm{C}=\mathrm{O}\right.$ st), 1224 (acetyl-C-O st); ${ }^{1} \mathrm{H}-$ NMR (300 MHz, $\left.\mathrm{CDCl}_{3}\right): \delta=1.24\left(\mathrm{~d}, J=6.3 \mathrm{~Hz}, 3 \mathrm{H}, 6-\mathrm{H}_{3 \alpha}\right), 1.30\left(\mathrm{~d}, J=6.1 \mathrm{~Hz}, 3 \mathrm{H}, 6-\mathrm{H}_{3 \beta}\right)$, $1.52\left(\mathrm{~m}, 2 \mathrm{H}, 3-\mathrm{H}_{2 \beta}\right), 1.82\left(\mathrm{~m}, 2 \mathrm{H}, 3-\mathrm{H}_{2 \alpha}\right), 2.03$ (s, $\left.3 \mathrm{H}, 1-\mathrm{OAc}_{\alpha}\right), 2.05$ (s, $\left.3 \mathrm{H}, 1-\mathrm{OAc}_{\beta}\right), 2.11(\mathrm{~s}$, $\left.3 \mathrm{H}, 2-\mathrm{OAc}_{\beta}\right), 2.16\left(\mathrm{~s}, 3 \mathrm{H}, 2-\mathrm{OAc}_{\alpha}\right), 2.23\left(\mathrm{~s}, 6 \mathrm{H}, \mathrm{N}\left(\mathrm{CH}_{3}\right)_{2 \beta}\right), 2.26\left(\mathrm{~s}, 6 \mathrm{H}, \mathrm{N}\left(\mathrm{CH}_{3}\right)_{2 \alpha}\right), 2.35-2.46$ $\left(\mathrm{m}, 1 \mathrm{H}+1 \mathrm{H}, 4-\mathrm{H}_{\alpha}, 4-\mathrm{H}_{\beta}\right), 3.67\left(\mathrm{dq}, J=9.6,6.1 \mathrm{~Hz}, 1 \mathrm{H}, 5-\mathrm{H}_{\beta}\right), 3.84(\mathrm{dq}, J=10.0,6.2 \mathrm{~Hz}, 1 \mathrm{H}$, $\left.5-\mathrm{H}_{\alpha}\right), 4.78\left(\mathrm{ddd}, J=11.4,8.2,5.2 \mathrm{~Hz}, 1 \mathrm{H}, 2-\mathrm{H}_{\beta}\right), 4.94\left(\mathrm{ddd}, J=12.1,5.0,3.4 \mathrm{~Hz}, 1 \mathrm{H}, 2-\mathrm{H}_{\alpha}\right)$, $5.59\left(\mathrm{~d}, J=8.2 \mathrm{~Hz}, 1 \mathrm{H}, 1-\mathrm{H}_{\beta}\right), 6.13\left(\mathrm{~d}, J=3.4 \mathrm{~Hz}, 1 \mathrm{H}, 1-\mathrm{H}_{\alpha}\right) ;{ }^{13} \mathrm{C}-\mathrm{NMR}\left(75.5 \mathrm{MHz}, \mathrm{CDCl}_{3}\right): \delta$ $=18.15\left(\mathrm{C}-6_{\mathrm{b}}\right), 18.18\left(\mathrm{C}-6_{\alpha}\right), 20.43^{*}, 20.82^{*}, 20.95^{*}, 21.01^{*}\left(4 \mathrm{CH}_{3} \mathrm{CO}\right), 40.60\left(\mathrm{~N}\left(\mathrm{CH}_{3}\right)_{2} \alpha+\beta\right)$, $63.80\left(\mathrm{C}-4_{\beta}\right), 63.88\left(\mathrm{C}-4_{\alpha}\right), 68.70\left(\mathrm{C}-5_{\alpha}\right), 68.98\left(\mathrm{C}-2_{\alpha}\right), 70.02\left(\mathrm{C}-2_{\beta}\right), 74.85\left(\mathrm{C}-5_{\beta}\right), 89.12\left(\mathrm{C}-1_{\alpha}\right)$, $93.50\left(\mathrm{C}-1_{\beta}\right), 169.45^{*}, 169.54^{*}, 169.82^{*}, 169.98^{*}\left(4 \mathrm{CH}_{3} \mathrm{CO}\right) ; \mathrm{MS}(\mathrm{EI}): \mathrm{m} / \mathrm{z}(\%)=259.2$ (14) $[\mathrm{M}]^{+}, 200.2$ (24) $[\mathrm{M}-\mathrm{OAc}]^{+}, 156.2$ (26) [M - OAc - $\left.\mathrm{N}\left(\mathrm{CH}_{3}\right)_{2}\right]^{+}$; HRMS-EI: Calcd. for $\mathrm{C}_{12} \mathrm{H}_{21} \mathrm{NO}_{5}$ : 259.1420. Found: 259.1420.

(4S)-2-O-Acetyl-3,6-dideoxy-4-dimethylamino- $\alpha / \beta$-D-xylo-hexopyranose,

(2R)-2-Acetoxy$\boldsymbol{\alpha} / \boldsymbol{\beta}$-D-forosamine (4). A solution of $11(25.2 \mathrm{mg}, 97.2 \mu \mathrm{mol})$ and ethanolamine $(58 \mu \mathrm{L}$, $0.97 \mathrm{mmol}, 10$ equiv.) in ethyl acetate $(5 \mathrm{ml})$ was stirred for $3.5 \mathrm{~d}$ at room temperature. Then the reaction mixture was purified directly by column chromatography $\left(\mathrm{CH}_{2} \mathrm{Cl}_{2} /\right.$ methanol 7:1) to give $4(15.4 \mathrm{mg}, 70.9 \mu \mathrm{mol}, 73 \%)$ as a colourless oil; $R_{\mathrm{f}}=0.20$ (n-pentane/ethyl acetate/methanol 7:2:1); IR ( $\left.\mathrm{NaCl}, \mathrm{cm}^{-1}\right)$ : 3100-3600 (OH), 2972, 2936, 1739 (C=O st), 1243 (acetyl-C-O st), 1157, 1098, 1059, 1033, 963; ${ }^{1} \mathrm{H}-\mathrm{NMR}\left(300 \mathrm{MHz}, \mathrm{CDCl}_{3}\right): \delta=1.21$ (d, J=6.2 Hz, $3 \mathrm{H}, 6-\mathrm{H}_{3 \alpha}$ ), $1.30\left(\mathrm{~d}, J=6.0 \mathrm{~Hz}, 3 \mathrm{H}, 6-\mathrm{H}_{3 \beta}\right), 1.52$ ('q', $\left.J=11.9 \mathrm{~Hz}, 2 \mathrm{H}, 3-\mathrm{H}_{2 \beta}\right), 1.86$ ('q', J=11.8 Hz, $2 \mathrm{H}$, 
3- $\left.\mathrm{H}_{2 \alpha}\right), 2.075\left(\mathrm{~s}, 3 \mathrm{H}, \mathrm{OAc}_{\alpha / \beta}\right), 2.077\left(\mathrm{~s}, 3 \mathrm{H}, \mathrm{OAc}_{\beta / \alpha}\right), 2.123\left(\mathrm{~s}, 6 \mathrm{H}, \mathrm{N}\left(\mathrm{CH}_{3}\right)_{2 \alpha / \beta}\right), 2.23(\mathrm{~s}, 6 \mathrm{H}$, $\left.\mathrm{N}\left(\mathrm{CH}_{3}\right)_{2 \beta / \alpha}\right), 2.30-2.40\left(\mathrm{~m}, 1 \mathrm{H}+1 \mathrm{H}, 4-\mathrm{H}_{\alpha}, 4-\mathrm{H}_{\beta}\right), 3.53\left(\mathrm{dq}, J=9.5,6.0 \mathrm{~Hz}, 1 \mathrm{H}, 5-\mathrm{H}_{\beta}\right), 4.01$ $\left(\mathrm{dq}, J=9.7,6.2 \mathrm{~Hz}, 1 \mathrm{H}, 5-\mathrm{H}_{\alpha}\right), 4.53\left(\mathrm{~d}, J=7.9 \mathrm{~Hz}, 1 \mathrm{H}, 1-\mathrm{H}_{\beta}\right), 4.60$ (ddd, $J=11.5,7.9,5.0 \mathrm{~Hz}$, $\left.1 \mathrm{H}, 2-\mathrm{H}_{\beta}\right), 4.90\left(\mathrm{ddd}, J=11.7,5.1,3.5 \mathrm{~Hz}, 1 \mathrm{H}, 2-\mathrm{H}_{\alpha}\right), 5.20\left(\mathrm{~d}, J=3.5 \mathrm{~Hz}, 1 \mathrm{H}, 1-\mathrm{H}_{\alpha}\right) ;{ }^{13} \mathrm{C}-$ NMR (75.5 MHz, $\left.\mathrm{CDCl}_{3}\right): \delta=18.28^{*}, 18.33^{*}\left(\mathrm{C}-6_{\alpha}, \mathrm{C}-6_{\beta}\right), 19.81\left(\mathrm{C}-3_{\alpha}\right), 21.13\left(2 \mathrm{CH}_{3} \mathrm{CO}\right)$, $24.46\left(\mathrm{C}-3_{\beta}\right), 40.67\left(\mathrm{~N}\left(\mathrm{CH}_{3}\right)_{2} \alpha+\beta\right), 64.34^{*}, 64.41^{*}(\mathrm{C}-4 \alpha+\beta), 65.87 *, 70.81^{*}, 73.05^{*}, 74.12^{*}$ $\left(\mathrm{C}-2_{\alpha}, \mathrm{C}-2_{\beta}, \mathrm{C}-5_{\alpha}, \mathrm{C}-5_{\beta}\right), 89.54\left(\mathrm{C}-1_{\alpha}\right), 96.97\left(\mathrm{C}-1_{\beta}\right), 170.33^{*}, 171.24^{*}\left(\mathrm{CH}_{3} \mathrm{CO} \alpha+\beta\right)$; MS (ESI): $\mathrm{m} / \mathrm{z}(\%)=457.2(100)[2 \mathrm{M}+\mathrm{Na}]^{+}, 240.2(68)[\mathrm{M}+\mathrm{Na}]^{+}, 218.2(11)[\mathrm{M}+\mathrm{H}]^{+}, 200.2(9)$ $\left[\mathrm{M}-\mathrm{H}_{2} \mathrm{O}\right]^{+} ; \mathrm{C}_{10} \mathrm{H}_{19} \mathrm{NO}_{4}$ (217.26); HRMS-ESI: $[\mathrm{M}+\mathrm{H}]^{+}$Calcd. for $\mathrm{C}_{10} \mathrm{H}_{19} \mathrm{NO}_{4}$ : 218.13868 . Found: 218.13879.

\section{(4S)-2-O-Acetyl-3,6-dideoxy-4-dimethylamino- $\alpha / \beta$-D-xylo-hexopyranosyl}

trichloroacetimidate, (2R)-2-Acetoxy- $\alpha / \beta$-D-forosaminyl trichloroacetimidate (12). To a solution of $4(21.1 \mathrm{mg}, 97.1 \mu \mathrm{mol})$ in dry dichloro-methane $(3 \mu \mathrm{mL})$ were added at room temperature trichloroacetonitrile $(41.5 \mu \mathrm{L}, 486 \mu \mathrm{mol}, 5.0$ equiv.) and then slowly polymer supported DBU (108 mg, $48.6 \mu \mathrm{mol}$, loading $0.45 \mathrm{mmol} / \mathrm{g}, 0.5$ equiv.), and the mixture stirred for $2 \mathrm{~h}$ at room temperature. The reaction mixture was filtered and the resin was washed with dichloromethane $(30 \mathrm{~mL})$. The filtrate was concentrated under vacuo to give $\mathbf{1 2}$ as a yellowish oil $(24.1 \mathrm{mg}, 73.1 \mu \mathrm{mol}, 75 \%) ; R_{\mathrm{f}}=0.82$ (ethyl acetate, Alox N); ${ }^{1} \mathrm{H}-\mathrm{NMR}\left(600 \mathrm{MHz}, \mathrm{CDCl}_{3}\right): \delta=$ $1.10\left(\mathrm{~d}, J=6.1 \mathrm{~Hz}, 3 \mathrm{H}, 6-\mathrm{H}_{3 \alpha / \beta}\right), 1.16\left(\mathrm{~d}, J=6.1 \mathrm{~Hz}, 3 \mathrm{H}, 6-\mathrm{H}_{3 \beta / \alpha}\right), 1.98-2.20(\mathrm{~m}, 12 \mathrm{H}+12 \mathrm{H}$, $\mathrm{N}\left(\mathrm{CH}_{3}\right)_{2} \alpha+\beta$, OAc $\left.\alpha+\beta, 4-\mathrm{H} \alpha+\beta, 3-\mathrm{H}_{2} \alpha+\beta\right), 2.87$ (br s, $\left.1 \mathrm{H}+1 \mathrm{H}, \mathrm{C}=\mathrm{NH} \alpha+\beta\right), 3.54$ (m, $\left.1 \mathrm{H}, 5-\mathrm{H}_{\alpha}\right), 3.99\left(\mathrm{dq}, J=10.0,6.1 \mathrm{~Hz}, 1 \mathrm{H}, 5-\mathrm{H}_{\beta}\right), 4.88\left(\mathrm{ddd}, J=11.2,8.3,5.3 \mathrm{~Hz}, 1 \mathrm{H}, 2-\mathrm{H}_{\beta}\right)$, $5.02\left(\mathrm{ddd}, J=12.0,5.1,3.4 \mathrm{~Hz}, 1 \mathrm{H}, 2-\mathrm{H}_{\alpha}\right), 5.82\left(\mathrm{~d}, J=8.3 \mathrm{~Hz}, 1 \mathrm{H}, 1-\mathrm{H}_{\beta}\right), 6.16(\mathrm{~d}, J=3.4 \mathrm{~Hz}$, $\left.1 \mathrm{H}, 1-\mathrm{H}_{\alpha}\right)$.

Isopropyl 2-O-acetyl-3,6-dideoxy-4-dimethylamino- $\alpha / \beta$-D-xylo-hexopyranoside, Isopropyl (2R)-2-acetoxy- $\alpha / \boldsymbol{\beta}$ - D-forosaminopyranose (13/14). A solution of 2-propanol (33.3 $\mu \mathrm{L}$, $435 \mu \mathrm{mol}, 6.7$ equiv.) and acetonitrile $(9.1 \mu \mathrm{L}, 174 \mu \mathrm{mol}, 2.7$ equiv.) in dry dichloromethane (5 $\mathrm{mL})$ was stirred for $30 \mathrm{~min}$ over molecular sieves $(3 \AA)$ at $0{ }^{\circ} \mathrm{C}$. To this mixture a solution of 12 $(21.5 \mathrm{mg}, 65.3 \mu \mathrm{mol})$ in dry dichloromethane $(1 \mathrm{~mL})$ and then slowly a solution of $\mathrm{BF}_{3} \cdot \mathrm{OEt}_{2}$ $(27.6 \mu \mathrm{L}, 218 \mu \mathrm{mol}, 3.3$ equiv.) in dry dichloromethane $(1 \mathrm{~mL})$ was added. After stirring for 30 $\min$ at $0{ }^{\circ} \mathrm{C}$ and $1 \mathrm{~h}$ at room temperature the reaction was quenched by addition of triethylamine $(0.5 \mathrm{~mL})$ and the solvent removed under reduced pressure. Purification of the residue by column chromatography (n-pentane/ethyl acetate/methanol 7.5:2:0.5) gave a 1:3 mixture of 13 and 14 (13.2 $\mathrm{mg}, 50.9 \mu \mathrm{mol}, 78 \%$ ) as a yellowish solid as, which contained about $5 \%$ of trichloroacetamide; $R_{\mathrm{f}}=$ (n-pentane/ethyl acetate/methanol 7.5:2:0.5): 0.41/0.44; ${ }^{1} \mathrm{H}-\mathrm{NMR}(600$ $\left.\mathrm{MHz}, \mathrm{CDCl}_{3}\right): \delta=1.10-1.30\left(\mathrm{~m}, 9 \mathrm{H}+9 \mathrm{H}, 6-\mathrm{H}_{3}, \mathrm{CH}\left(\mathrm{CH}_{3}\right)_{2} \alpha+\beta\right), 1.50-1.80(\mathrm{~m}, 2 \mathrm{H}+2 \mathrm{H}, 3-$ $\left.\mathrm{H}_{2} \alpha+\beta\right), 2.03\left(\mathrm{~s}, 3 \mathrm{H}, \mathrm{OAc}_{\beta}\right), 2.05\left(3 \mathrm{H}, \mathrm{OAc}_{\alpha}\right), 2.25$ (br s, $\left.6 \mathrm{H}+6 \mathrm{H}, \mathrm{N}\left(\mathrm{CH}_{3}\right)_{2} \alpha+\beta\right), 2.40-$ $2.45(\mathrm{~m}, 1 \mathrm{H}+1 \mathrm{H}, 4-\mathrm{H} \alpha+\beta), 3.40-3.70\left(\mathrm{~m}, 1 \mathrm{H}+2 \mathrm{H}, 5-\mathrm{H} \alpha+\beta, \mathrm{CH}\left(\mathrm{CH}_{3}\right)_{2 \alpha}\right), 3.90$ (sept., $J=$ $\left.6.3 \mathrm{~Hz}, 1 \mathrm{H}, \mathrm{CH}\left(\mathrm{CH}_{3}\right)_{2 \beta}\right), 4.36\left(\mathrm{~d}, J=7.9 \mathrm{~Hz}, 1 \mathrm{H}, 1-\mathrm{H}_{\beta}\right), 4.60(\mathrm{ddd}, J=11.2,7.9,5.3 \mathrm{~Hz}, 1 \mathrm{H}, 2-$ $\left.\mathrm{H}_{\beta}\right), 4.75\left(\mathrm{~m}, 1 \mathrm{H}, 2-\mathrm{H}_{\alpha}\right), 4.90\left(\mathrm{~d}, J=3.5 \mathrm{~Hz}, 1 \mathrm{H}, 1-\mathrm{H}_{\alpha}\right) ; \mathrm{MS}(\mathrm{ESI}): \mathrm{m} / \mathrm{z}(\%)=282.1(48)[\mathrm{M}+$ 
$\mathrm{Na}]^{+}$, $260.2(100)[\mathrm{M}+\mathrm{H}]^{+}$; HRMS-ESI: $[\mathrm{M}+\mathrm{H}]^{+}$Calcd. for $\mathrm{C}_{13} \mathrm{H}_{25} \mathrm{NO}_{4}: 260.18563$. Found: 260.18587 .

\section{Acknowledgements}

We thank the Deutsche Forschungsgemeinschaft (SFB 416) and the Fonds der Chemischen Industrie for generous support.

\section{References and Footnotes}

1. (a) Mertz, F. P.; Yao, R. C. Int. J. System. Bacteriol. 1990, 40, 30. (b) Kirst, H. A.; Michel, K. H.; Martin, J. W.; Creemer, L. C.; Chio, E. H.; Yao, R. C., Nakatsukasa, W. M.; Boek, L. D.; Occolowitz, J. L.; Paschal, J. W.; Deeter, J. B.; Jones, N. D.; Thompson, G. D. Tetrahedron Lett. 1991, 32, 4839. (c) Boeck, L. D.; Chio, E. H.; Eaton, T. E.; Godfrey, O. W.; Michel, K. H.; Nakatsukasa, W. M.; Yao, R. C. Eur. Pat. Appl. EP 1990, 375316; Chem. Abstr. 1991, 114, 80066.

2. (a) Salgado, V. L.; Sparks; T. C. Comprehensive Molecular Insect Science 2005, 6, 137. (b) Salgado, V. L. Pestic. Biochem. Physiol. 1998, 60, 91. (c) Salgado, V. L. ; Sheets, J. J.; Watson, G. B.; Schmidt, A. L. Pestic. Biochem. Physiol. 1998, 60, 103.

3. (a) Moulton, J. K.; Pepper, D. A.; Dennehy, T. M. Pest. Manag. Sci. 2000, 56, 842. (b) Mau, R. F. L.; Gusukuma-Minuto, L. Proceedings of 5th International Seminar on Technology of Cole Crops Production, University de Celaya, Guanajuato, Mexico 2001, 75.

4. (a) Evans, D. A.; Black, W. C. J. Am. Chem. Soc. 1993, 115, 4497. (b) Paquette, L. A.; Gao, Z.; Ni, Z.; Smith, G. F. J. Am. Chem. Soc. 1998, 120, 2543. (c) Paquette, L. A.; Collado, I.; Purdie, M. J. Am. Chem. Soc. 1998, 120, 2553. (d) Mergott, D. J.; Frank, S. A.; Roush, W. R. PNAS 2004, 101, 33, 11955.

5. Yu, H. N.; Zhang, P.; Ling, C.-Ch.; Bundle, D. R. Tetrahedron: Asymmetry 2000, 11, 465.

6. Gotoh, M.; Kovac, P. J. Carbohydrate Chem. 1994, 13, 1193.

7. Bowers, S. E.; Mahmud, T.; Floss, H. G. Carbohydrate Res. 2002, 337, 297.

8. Tsvetkov, Y. E.; Shashkov, A. S.; Knirel, Y. A.; Zähringer, U. Carbohydrate Res. 2001, 335, 221.

9. Eis, M. J.; Ganem, B. Carbohydrate Res. 1988, 176, 316.

10. Hennen, W. J.; Sweers, H. M.; Wang, Y.-F.; Wong, C.-H. J. Org. Chem. 1988, 53, 4939.

11. Malik, A.; Afza, N.; Voelter, W. Liebigs Ann. Chem. 1984, 4, 636.

12. Vogel, C.; Bergemann, C.; Boye, H.; Ott, A. Y.; Betaneli, V. I.; Kochetkov, N. K.; J. Carbohydrate Chem. 1994, 13, 47. 
13. (a) Slaghek, T. M. Carbohydrate Res. 1990, 207, 237. (b) Han, X.-B.; Jiang, Z.-H. Schmidt, R. R. Liebigs Ann. Chem. 1993, 853. (c) Jiang, Z.-H.; Schmidt, R. R. Liebigs Ann. Chem. 1992, 975. (d) Khare, N. K.; Sood, R. K.; Chatterjee, D.; Rivoire, B.; Brennan, P. Carbohydrate Res. 1991, 216, 357. (e) Kerekgyarto, J.; Szurmai, Z.; Liptak, A. Carbohydrate Res. 1993, 245, 65.

14. Ichikawa, Y.; Sim, M. M.; Wong, C.-H. J. Org. Chem. 1992, 57, 2943.

15. Hennen, W. J.; Sweers, H. M.; Wang, Y.-F.; Wong, C.-H. J. Org. Chem. 1988, 53, 4939.

16. (a) Ariosa-Alvarez, A.; Arenciba-Mohar, A.; Madrazo-Alonso, O.; Garciá-Imia, L.; SierraGonzalez, G.; Verez-Bencomo, V. J. Carbohydr. Chem. 1998, 17, 1307. (b) Palomino, J. C. C.; Rensoli, M. H.; Verez-Bencomo, V. J. Carbohydr. Chem. 1996, 15, 137.

17. (a) Chiara, J. L.; Encinas, L.; Díaz B. Tetrahedron Lett. 2005, 46, 2445. (b) Tomoi, M.; Kato, Y.; Kakiuchi, H. Macromol. Chem. 1984, 185, 2117.

18. (a) Schmidt, R. R.; Michel, J. Angew. Chem. 1980, 92, 763. (b) Schmidt, R. R. Angew. Chem. 1986, 98, 213. 